\title{
Anderson Localization versus Delocalization of Interacting Fermions in One Dimension
}

\author{
P. Schmitteckert, ${ }^{1}$ T. Schulze, ${ }^{2}$ C. Schuster, ${ }^{2}$ P. Schwab, ${ }^{2,3}$ and U. Eckern ${ }^{2}$ \\ ${ }^{1}$ IPCMS-GEMME, CNRS, 23 rue du Loss, F-67307 Strasbourg Cedex, France \\ ${ }^{2}$ Institut für Physik, Universität Augsburg, D-86135 Augsburg, Germany \\ ${ }^{3}$ Dipartimento di Fisica, Università “La Sapienza,” Piazzale A. Moro 2, I-00185, Roma, Italy \\ (Received 9 June 1997)
}

\begin{abstract}
Using the density matrix renormalization group algorithm, we investigate the lattice model for spinless fermions in one dimension in the presence of a strong interaction and disorder. The phase sensitivity of the ground state energy is determined with high accuracy for systems up to a size of 60 lattice constants. This quantity is found to be log normally distributed. The fluctuations grow algebraically with system size with a universal exponent of $\approx 2 / 3$ in the localized region of the phase diagram. Surprisingly we find, for an attractive interaction, a delocalized phase of finite extension. The boundary of this delocalized phase is determined. [S0031-9007(97)05095-3]
\end{abstract}

PACS numbers: 71.30. $+\mathrm{h}, 72.15 . \mathrm{Rn}$

The influence of electron-electron interaction on Anderson localization has attracted a lot of interest for several years. Many recent theoretical studies [1-5] were motivated by the experimental observation [6] of persistent currents in mesoscopic rings. So far, however, theory has not been successful in explaining the magnitude of the effect, even though both interaction and disorder have been considered for the experimental situation of a large number of transverse channels. For this case, the accuracy of theoretical approaches is rather limited. But even in the case of one dimension, certainly not appropriate for the experiment, detailed results are available only for interacting systems in the absence of disorder [7-9], or for disordered systems in the absence of interactions [10]. However, a clear understanding of the interplay between interaction and disorder has not yet been obtained.

In this Letter, we present novel results of a detailed, quantitative study of a simple interacting-fermion model with disorder. We determine the phase sensitivity of the ground state energy, i.e., its dependence on boundary conditions, with high accuracy for a wide range of parameters and system sizes up to 60 lattice constants. Our main results are (i) a universal behavior of the rms value of the logarithmic phase sensitivity, which grows with system size $M$ proportional to $M^{2 / 3}$ in the localized region; and (ii) the zero-temperature phase diagram, which shows, for an attractive interaction, a delocalized phase of finite extension.

The numerical results are obtained with the density matrix renormalization group algorithm (DMRG) [11], which allows the calculation of ground state properties of disordered, interacting-fermion systems with an accuracy which is comparable to exact diagonalization, but for much larger systems [12,13]. In our implementation of the DMRG we perform five finite lattice sweeps, keeping up to 750 states per block.

We consider a chain of spinless fermions with nearestneighbor interaction and disorder,

$$
\begin{aligned}
H= & -t \sum_{i}\left(c_{i}^{+} c_{i+1}+c_{i+1}^{+} c_{i}\right)+\sum_{i} \epsilon_{i} c_{i}^{+} c_{i} \\
& +V \sum_{i} n_{i} n_{i+1},
\end{aligned}
$$

and twisted boundary conditions, $c_{0}=e^{i \phi} c_{M}$. The length of the chain is denoted by $M$, and the particle number is $N$. For simplicity, we will set $t=1$ in some of the formulas below.

The ground state energy $E(\phi)$ depends on the phase $\phi$. The energy difference between periodic and antiperiodic boundary conditions, $\Delta E=(-)^{N}[E(0)-E(\pi)]$, the persistent current, $I(\phi) \sim-E^{\prime}(\phi)$, and the charge stiffness, $D \sim E^{\prime \prime}(\phi=0)$, are a measure of the phase sensitivity of the system. In the clean limit, i.e., $\epsilon_{n}=0$ for all $n$, the ground state energy can be determined from the Bethe ansatz [7,14]. At half-filling, the phase sensitivity in the limit of large systems $(M \rightarrow \infty)$ is given by [15]

$$
E_{M}(\phi)-M \epsilon_{\infty}=-\frac{\pi v}{6 M}\left(1-3 K \frac{\phi^{2}}{\pi^{2}}\right),
$$

where $E_{M}$ is the ground state energy of the $M$-site system, $\epsilon_{\infty}$ is the energy density in the thermodynamic limit, and $v$ is the Fermi velocity, $v=\pi t \sin (2 \eta) /(\pi-2 \eta)$. The interaction parameter is $K=\pi / 4 \eta$, where $\eta$ parametrizes the interaction according to $V=-2 t \cos (2 \eta)$. Thus $M \Delta E=\pi v K / 2$.

For the noninteracting system, the phase sensitivity in the presence of a single defect $\left(\epsilon_{0} \neq 0\right)$ can be determined easily [16]. In the presence of both electron-electron interaction and defect, it is more difficult to calculate $\Delta E$. However, it is known that the ground state and the low-lying excitations can be described within the framework of a Luttinger liquid [9,17]. Combining a firstorder perturbative calculation with the scaling equations of Kane and Fisher [18], we find in the case of a weak impurity

$$
M \Delta E=\frac{\pi v K}{2}-\left|\epsilon_{0}\right|\left(\frac{M}{M_{0}}\right)^{1-K}
$$


where $M_{0}$ is a short distance cutoff which is-for the half-filled band-of the order of the lattice spacing. Using a duality relation between a weak impurity and a weak link [18], we obtain for a strong impurity

$$
M \Delta E=\frac{4 t^{2}}{\left|\epsilon_{0}\right|}\left(\frac{M}{M_{0}}\right)^{1-1 / K},
$$

since the transmission through a strong defect is proportional to $t^{2} / \epsilon_{0}$. Figure 1 shows $M \Delta E$ as a function of interaction for system sizes $M=60$ and several defect strengths. The points are numerical results from the DMRG, the full lines are analytical results from the equations above. The cutoff parameter $M_{0}$ was fitted in order to obtain agreement between numerical and analytical results, giving $M_{0} \approx 2$. An attractive interaction makes the barrier more transparent, while a repulsive interaction increases the defect strength. Deviations from the analytical results are found when the "strong" impurity becomes so weak that a first-order expansion is no longer appropriate. In addition, we find further deviations near $V= \pm 2$, where the Luttinger liquid becomes unstable. At $V=-2$, there is an instability with respect to phase separation $(v \rightarrow 0, K \rightarrow \infty)$. At $V=+2$, there is an instability to formation of a charge-density wave, since at this point $4 k_{F}$-backscattering processes become relevant.

In the presence of a weak random potential, but $V=0$, we find, generalizing the single impurity result,

$$
M \Delta E=\pi t-\left|\sum_{n=1}^{M} \epsilon_{n} e^{2 i k_{F} n}\right|,
$$

where we introduce disorder by taking the $\left\{\epsilon_{n}\right\}$ uniformly random distributed over the interval $[-W / 2, W / 2]$. At half-filling, $k_{F}=\pi / 2$, the sum $\sum_{n=1}^{M} \epsilon_{n}(-1)^{n}$ can be considered as a one-dimensional random walk with $M$ steps. Recalling that a random walk leads to a Gaussian distribution of distances, we obtain for the average phase

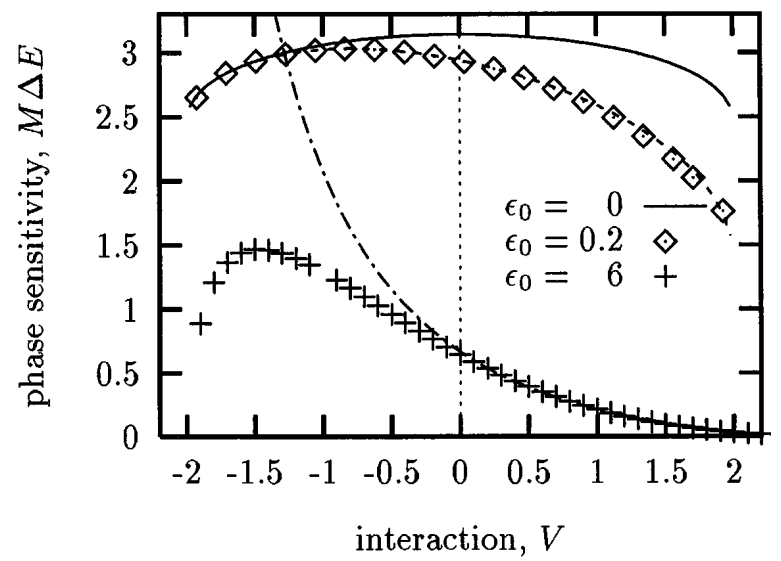

FIG. 1. Phase sensitivity of the ground state energy in the presence of a single defect as a function of interaction for several defect strengths $\epsilon_{0}$. The $\diamond$ and + points are numerical results (system size $M=60$ ). The solid, dashed, and dash-dotted lines are obtained from Eqs. (2), (3), and (4), respectively. sensitivity and the fluctuations

$$
\begin{gathered}
\langle M \Delta E\rangle=\pi t-\frac{W \sqrt{M}}{\sqrt{6 \pi}}, \\
\sigma_{M \Delta E}^{2}=M \frac{W^{2}}{12}\left(1-\frac{2}{\pi}\right) .
\end{gathered}
$$

The brackets \langle\rangle denote the impurity average. It is apparent in Eq. (6) that the perturbation theory breaks down for arbitrarily weak disorder, if the system is large enough: As is well known, in 1D even weak disorder leads to localization, with a localization length which is proportional to $t^{2} / W^{2}$. In large systems, $M \Delta E$ is drastically reduced due to disorder, however, numerical results (using exact diagonalization methods, which are straightforward as long as $V=0$ ) indicate that it remains positive for all realizations of the disorder [13], in agreement with a theorem by Leggett [19]. For large systems we find an exponential decay of the average phase sensitivity. In the localized regime, i.e., $M>\xi$, the logarithm of $M \Delta E$ has approximately a normal distribution [13]. From our numerical data, where we averaged over $10^{4}$ realizations of the disorder potential and considered systems of up to $10^{3}$ sites, we find for the average logarithmic phase sensitivity and its variance $\sigma^{2}$ in the limit of large systems $(M \rightarrow \infty)$ the following:

$$
\begin{aligned}
\langle\ln (M \Delta E)\rangle & \approx-\frac{M}{\xi}+0.76, \\
\sigma_{\ln (M \Delta E)} & \approx\left(\frac{0.52 M}{\xi}\right)^{2 / 3},
\end{aligned}
$$

with $\xi=114 t^{2} / W^{2}$. In order to check the universality of the exponent, we calculated the phase sensitivity for strong disorder up to $W=15 t$, and for different fillings. We always found the exponent $2 / 3$ in the localized region.

The interaction changes drastically some of the results described above. Applying the Kane-Fisher scaling to Eq. (5), we obtain

$$
M \Delta E=\frac{\pi v K}{2}-\left|\sum_{n=1}^{M} \epsilon_{n} e^{2 i k_{F} n}\right|\left(\frac{M}{M_{0}}\right)^{1-K}
$$

since the strength of each defect is renormalized. The average phase sensitivity is then given by

$$
\langle M \Delta E\rangle=\frac{\pi v K}{2}-\frac{W \sqrt{M_{0}}}{\sqrt{6 \pi}}\left(\frac{M}{M_{0}}\right)^{(3-2 K) / 2},
$$

and the fluctuations are

$$
\sigma_{M \Delta E}^{2}=\frac{W^{2} M_{0}}{12}\left(1-\frac{2}{\pi}\right)\left(\frac{M}{M_{0}}\right)^{3-2 K} .
$$

Again, a repulsive interaction tends to enhance the effective strength of the defects, and an attractive interaction reduces it. Especially, for $K>3 / 2$, i.e., $V<-1$, the strength of each defect vanishes so fast that disorder becomes an irrelevant perturbation; there is no localization [20,21]. We discuss the localized phase, $V>-1$, 


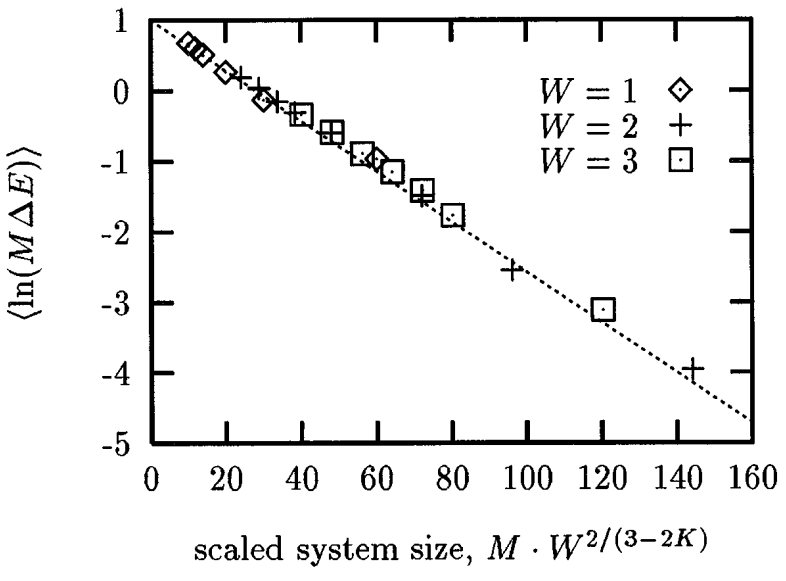

FIG. 2. Average logarithmic phase sensitivity as a function of the scaled system size, for $V=1.2$ and disorder $W=1,2,3$.

first. Assuming that only one relevant length scale exists, i.e., the localization length $\xi$, one concludes from (11) that $\xi \propto W^{2 /(2 K-3)}$ for weak disorder. This is verified in Fig. 2, where we plot the logarithmic phase sensitivity as a function of the scaled system size. In the case of the largest systems considered $(M=60)$, we averaged over several hundred realizations, whereas for short systems $(M<20)$ we used ensembles of more than $10^{3}$ realizations. With good accuracy, points corresponding to different strengths of disorder lie on the same curve, i.e., the localization length is indeed the only relevant scale, even for $M \gg \xi$, where the perturbation theory breaks down. The average phase sensitivity, shown in Fig. 2, is for large systems given approximately by $(V=1.2)$,

$$
\langle\ln (M \Delta E)\rangle=-M / \xi+1,
$$

with the localization length $\xi \approx 28 W^{-2 /(3-2 K)}$.

The rms value, $\sigma_{\ln (M \Delta E)}$, shown in Fig. 3, is for small systems proportional to $M^{(3-2 K) / 2}$ [see Eq. (12)]. (Note that $\sigma_{\ln (M \Delta E)}$ and $\sigma_{M \Delta E}$ are directly related to each other,

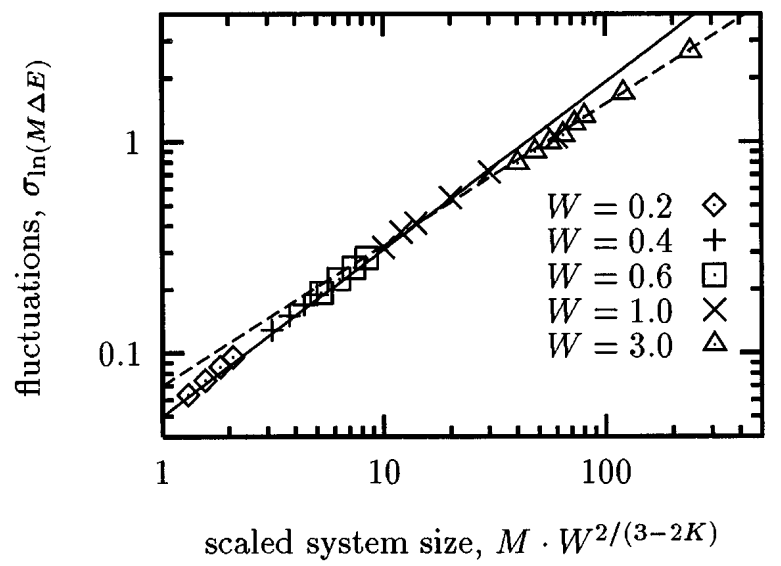

FIG. 3. Rms value of $\ln (M \Delta E)$ as a function of scaled system size (again, $V=1.2$ ). The full line is the analytic result according to Eq. (12), which explains the low- $M$ behavior. For large systems, $M>\xi, \sigma_{\ln (M \Delta E)}$ is proportional to $M^{2 / 3}$ (dashed line) as in the noninteracting case.

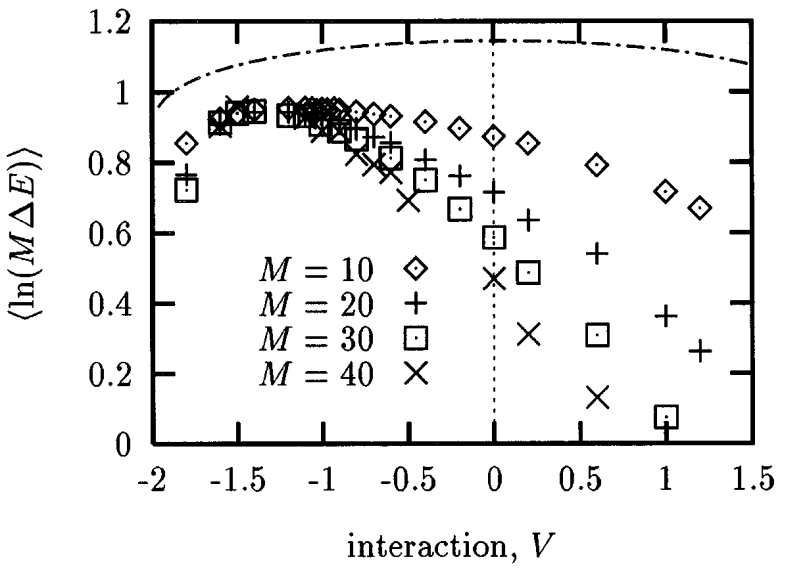

FIG. 4. Average logarithmic phase sensitivity, as a function of interaction. for system sizes ranging from 10 to $40 ; W=1$. For comparison, we included the result in the clean limit ( $W=0$, dash-dotted line).

provided $\sigma_{M \Delta E} \ll\langle M \Delta E\rangle$.) A crossover is apparent for $M \approx \xi$, i.e., when the fluctuations of $M \Delta E$ are comparable to its average. For large systems we again find the fluctuations to be proportional to $M^{2 / 3}$, as in the noninteracting limit. Explicitly, we found from our numerical data $(V=1.2$, i.e., $K \approx 0.71)$

$$
\sigma_{\ln (M \Delta E)} \approx 0.027\left(M W^{2 /(3-2 K)}\right)^{2 / 3} .
$$

In Fig. 4 we plot $\langle\ln (M \Delta E)\rangle$ as a function of interaction and for several system sizes (here $W=1$ ). For comparison, we included the phase sensitivity in the absence of disorder. Between $V \approx-1.6$ and $\approx-1.1$, the phase sensitivity remains almost unreduced, even for large systems. We believe that this region corresponds to the delocalized phase predicted earlier [20,21]. This assertion is confirmed by an apparent divergence of the localization length when approaching the phase boundary from the localized side [22]. Nevertheless, the phase sensitivity remains smaller than in the clean system since the parameters $v$ and $K$ scale downwards due to the random potential [21].

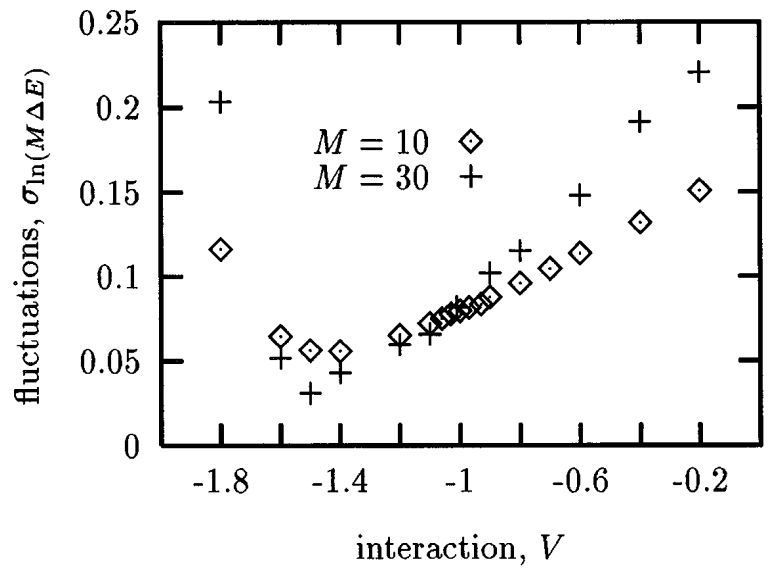

FIG. 5. Rms value of the logarithmic phase sensitivity versus interaction, for $M=10$ and $30 ; W=1$. 


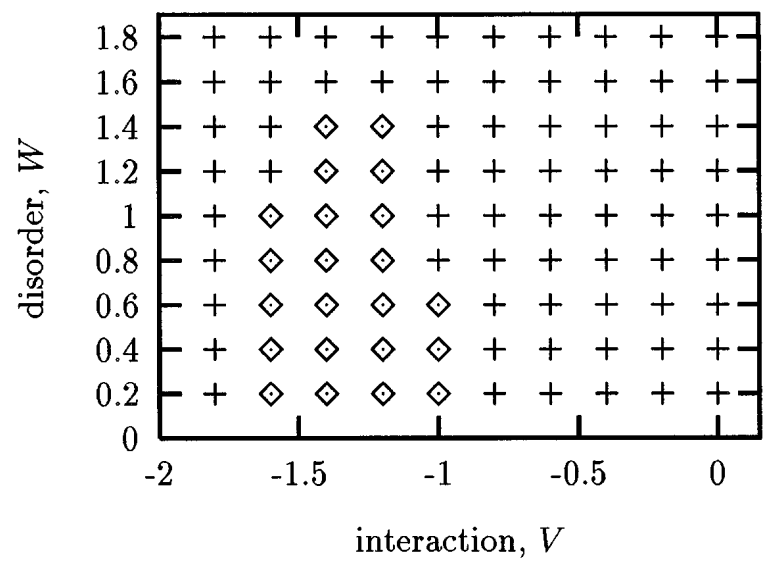

FIG. 6. Phase diagram. The symbol $\diamond(+)$ denotes the region where the variance of the logarithmic phase sensitivity decreases (increases) as a function of the system size. We considered up to 50 sites. The $\diamond$ region corresponds to a delocalized ground state.

The fluctuations of the logarithmic phase sensitivity provide another, more accurate method for determining the extension of the delocalized phase. Selected data are shown in Fig. 5 for $M=10$ and 30. As discussed in connection with Eq. (12), a decreasing variance (with increasing $M$, compare points $\diamond$ with + ) implies that the disorder scales to smaller values, hence the system is delocalized, while the variance increases for a localized ground state. Using this property as the criterion, we obtain, with considerable numerical effort, the phase diagram shown in Fig. 6. These results are based on system sizes between 30 and 50 sites. Clearly, by this method, we can only give a rough estimate of the phase boundary, and it is possible that we somewhat overestimate the size of the delocalized region.

In summary, using the DMRG algorithm, we have obtained high accuracy results for the ground state energy for a model of interacting fermions with disorder. In the weak disorder limit, we verified quantitatively several predictions on disordered Luttinger liquids. In the localized region, we determined the localization length and the distribution of the phase sensitivity. The latter is nearly log normally distributed, with a universal size dependence of the fluctuations proportional to $M^{2 / 3}$. We confirmed the existence of a delocalized region in the phase diagram. As far as we know, we are the first to give a quantitative estimate (see, however, Ref. [23]) of the size (as a function of disorder and interaction) of this region.

This work was supported by the Deutsche Forschungsgemeinschaft (Forschergruppe HO 955/2-1) and through the TMR program of the European Union (P. Schm. and P. Schw.). The calculations were partly done on the IBM SP2 at the Leibniz-Rechenzentrum in Munich.

[1] V. Ambegaokar and U. Eckern, Phys. Rev. Lett. 65, 381 (1990); 67, 3192 (1991); A. Schmid, ibid. 66, 80 (1991); ibid. 66, 1379 (1991).
[2] M. Abraham and R. Berkovits, Phys. Rev. Lett. 70, 1509 (1993); G. Bouzerar, D. Poilblanc, and G. Montambaux, Phys. Rev. B 49, 8258 (1994); M. Ramin, B. Reulet, and H. Bouchiat, Phys. Rev. B 51, 5582 (1995).

[3] T. Giamarchi and B. S. Shastry, Phys. Rev. B 51, 10915 (1995).

[4] H. Kato and D. Yoshioka, Phys. Rev. B 50, 4943 (1994).

[5] A. Cohen, R. Berkovits, and A. Heinrich, Int. J. Mod. Phys. B 11, 1845 (1997).

[6] L. P. Lévy, G. Dolan, J. Dunsmuir, and H. Bouchiat, Phys. Rev. Lett. 64, 2074 (1990); V. Chandrasekhar et al., Phys. Rev. Lett. 67, 3578 (1991); D. Mailly, C. Chapelier, and A. Benoit, Phys. Rev. Lett. 70, 2020 (1993); P. Mohanty, E. M. Q. Jariwala, M. B. Ketchen, and R. A. Webb, in Quantum Coherence and Decoherence, edited by K. Fujikawa and Y. A. Ono (Elsevier Science, Amsterdam, 1996), p. 191.

[7] C. J. Hamer, G. R.W. Quispel, and M. T. Batchelor, J. Phys. A 20, 5677 (1987).

[8] B. S. Shastry and B. Sutherland, Phys. Rev. Lett. 65, 243 (1990); B. Sutherland and B. S. Shastry, ibid. 65, 1833 (1990).

[9] D. Loss, Phys. Rev. Lett. 69, 343 (1992).

[10] A. A. Abrikosov and I. A. Ryzkhin, Adv. Phys. 27, 147 (1978); M. Kappus and F. J. Wegner, Z. Phys. B 45, 15 (1981); R. Kree and A. Schmid, Z. Phys. B 42, 297 (1981); G. Czycholl, B. Kramer, and A. MacKinnon, Z. Phys. B 43, 5 (1981).

[11] S. R. White, Phys. Rev. Lett. 69, 2863 (1992); S. R. White and R. M. Noack, Phys. Rev. Lett. 68, 3487 (1992); S. R. White, Phys. Rev. B 48, 10345 (1993).

[12] H. Pang, S. Liang, and J. F. Annett, Phys. Rev. Lett. 71, 4377 (1993).

[13] P. Schmitteckert and U. Eckern, Phys. Rev. B 53, 15397 (1996).

[14] F. Woynarovich and H.P. Eckle, J. Phys. A 20, L97 (1987).

[15] The ground state energy is periodic in $\phi$ with period $2 \pi$. Here we consider odd $N$, and the interval $-\pi \ldots \pi$.

[16] H.-F. Cheung, Y. Gefen, E. K. Riedel, and W.-H. Shih, Phys. Rev. B 37, 6050 (1988).

[17] A. O. Gogolin and N. V. Prokof'ev, Phys. Rev. B 50, 4921 (1994).

[18] C. L. Kane and M. P. A. Fisher, Phys. Rev. Lett. 68, 1220 (1992).

[19] A.J. Leggett, in Granular Nanoelectronics, edited by D. K. Ferry (Plenum, New York, 1991), p. 297.

[20] W. Apel and T. M. Rice, Phys. Rev. B 26, 7063 (1982).

[21] T. Giamarchi and H. J. Schulz, Phys. Rev. B 37, 325 (1988).

[22] T. Schulze, Diplom thesis, Universität Augsburg, 1997 (unpublished).

[23] After submission of the manuscript, we were informed by G. Bouzerar of an earlier attempt [G. Bouzerar and D. Poilblanc, J. Phys. I (France) 4, 1699 (1994)] to estimate the delocalized region on the basis of the renormalization group equations. This procedure overestimates the region of extended states by about a factor of 4 . Note that our convention for the hopping matrix element $t$ differs from the one in that paper. We are grateful for this reference. 